\title{
Using the Oceans as a Calorimeter to Quantify the Solar Radiative Forcing
}

\author{
Nir J. Shaviv \\ Racah Institute of Physics, Hebrew University of Jerusalem, Giv'at Ram, Jerusalem 91904, Israel
}

\begin{abstract}
.
Over the 11-year solar cycle, small changes in the total solar irradiance (TSI) give rise to small variations in the global energy budget. It was suggested, however, that different mechanisms could amplify solar activity variations to give large climatic effects, a possibility which is still a subject of debate. With this in mind, we use the oceans as a calorimeter to measure the radiative forcing variations associated with the solar cycle. This is achieved through the study of three independent records, the net heat flux into the oceans over 5 decades, the sea level change rate based on tide gauge records over the $20^{\text {th }}$ century, and the sea surface temperature variations. Each of the records can be used to consistently derive the same oceanic heat flux. We find that the total radiative forcing associated with solar cycles variations is about 5 to 7 times larger than just those associated with the TSI variations, thus implying the necessary existence of an amplification mechanism, though without pointing to which one.
\end{abstract}

\section{Introduction}

Over the 11-year solar cycle, the "solar constant" of roughly $1366 \mathrm{~W} / \mathrm{m}^{2}$ varies by about $1 \mathrm{~W} / \mathrm{m}^{2}$, which is equivalent to $\sim 0.17 \mathrm{~W} / \mathrm{m}^{2}$ on top of the $\sim 240 \mathrm{~W} / \mathrm{m}^{2}$ global average of the non-reflected component. This is a very small contribution to the net radiative forcing [Foukal et al., 2006]. Nonetheless, various climatic variations synchronized with solar variations do exist, whether over the solar cycle [van Loon and Labitzke, 2000; White et al., 1997; Svensmark, 1998; Marsh and Svensmark, 2000b; Shaviv, 2005; Douglass and Clader, 2002], or over longer time scales [Eddy, 1976; Neff et al., 2001; Solanki and Fligge, 2002; Bond et al., 2001]

Two possibilities, or a combination of them, can explain the large climatic variations observed. The climate system could simply be very sensitive to any changes in the radiative forcing. A sensitivity of $\sim 0.6^{\circ} \mathrm{C} /\left(\mathrm{W} / \mathrm{m}^{2}\right)$ for $11-\mathrm{yr}$ signals (or several times larger for the equilibrium sensitivity) would allow the small TSI variations to explain the observed temperature variations [e.g., Shaviv, 2005]. Alternatively, the large non-thermal solar activity variations could be amplified by a mechanism unrelated to the solar irradiance. Examples include hyper-sensitivity to UV [Haigh, 1994] and the solar-wind modulated cosmic ray flux (CRF) [Ney, 1959; Svensmark, 1998]. Clearly, a measurement of the actual variations in the radiation budget should prove interesting, verifying or refuting the existence of an indirect mechanism.

We begin with a theoretical analysis relating the different data sets we use, that of the ocean heat content, the sea level change rate and the sea surface temperature. We then continue with reconstructing the solar driven quasi-decadal oceanic heat content variations, either directly from the heat content data set, or indirectly from the two others. In other words, we use the oceans as a calorimeter to measure the radiative imbalance associated with the solar cycle. This imbalance will be shown to be large, thus implying that a large amplification mechanism is necessarily operating.

\section{Theoretical and empirical relations}

Our main goal is to obtain the radiative forcing associated with the solar activity, through the estimate of the flux going into and out of the oceans every solar cycle. We use three different datas sets: The Ocean Heat Content (OHC), the Sea Surface Temperature (SST) and the Sea Level Rate of change (SLR). Since only the first data set can directly yield the heat flux (through the time derivative of the heat content), we require some theoretical understanding to transform the other two sets into a heat flux. In $\S 2.1$, we derive a model based theoretical relation between the $\mathrm{OHC}$ and the SST, while we find an empirical relation between the SLR and $\mathrm{OHC}$ in $\S 2.2$.

Note that because deriving the OHC from the SST or from the SLR is less direct than using the actual OHC measurement, using the SST and SLR may introduce several systematic errors, as elaborated below. It is for this reason that the radiative forcing that we will derive below is going to be based only on the directly measured OHC. The two other data sets are very important, nonetheless, because they provide valuable consistency checks with a much higher signal to noise ratio than present in the OHC.

\subsection{The 1D model for the SST/OHC relation}

We assume that the ocean can be divided into a mixed layer just underneath the surface of the ocean, which exhibits very fast mixing, and the "deep ocean", where vertical diffusion is slow. Because of the fast vertical mixing in the mixed layer (ML), its temperature profile can be taken as uniform. To obtain the temperature profile below the ML, we need to solve the diffusion equation. Thus, our 1D model is as described in fig. 1.

Conservation of energy in the top layer implies:

$$
\ell C_{p} \frac{\partial \Delta T_{\mathrm{ML}}}{\partial t}=q_{\mathrm{top}}-q_{\mathrm{bottom}}
$$

where we work with temperature perturbations around the steady state. Also, $C_{p}$ is the heat capacity per unit volume at constant pressure, while $q_{t o p}$ is the net energy flux going into the mixed layer from the top. It includes the flux directly associated with the solar activity (which we wish to quantify), plus corrections due to the climate's response to the changed ocean temperature, which include two terms (in the linear limit). The first is heat loss due to the SST while the second is absorption depending on the temperature of the bottom of the atmosphere. Namely,

$$
q_{\mathrm{top}}=q_{\mathrm{top}, 0}-\left(\lambda_{o} \Delta T_{\mathrm{ML}}-\lambda_{a} \Delta T_{\mathrm{air}}\right) .
$$


Note that generally $\lambda_{o} \neq \lambda_{a}$ because either can independently interact with other heat reservoirs (e.g., short wavelength radiation and space).

Due to the small heat capacity of the atmosphere and land, we assume that it takes a short time for the atmospheric climate system above the oceans to reach an equilibrium with the oceans. Practically, there is therefore a negligible lag between the global air temperature and ocean temperature over the 11-year scale, thereby allowing us to assume that $\Delta T_{\text {air }}=\beta \Delta T_{\mathrm{ML}}$. The factor $\beta$ is not necessarily unity because the equilibrium change in the ocean temperature need not be the same as that of the air.

Thus, the atmospheric flux into the ocean depends on the ocean temperature, with an effective feedback parameter $\lambda_{a, \text { eff }}: \lambda_{a} \Delta T_{\text {air }} \equiv \lambda_{a} \beta \Delta T_{\mathrm{ML}} \equiv \lambda_{a, \text { eff }} \Delta T_{\mathrm{ML}}$. Eq. 2 , can be therefore written as

$$
q_{\mathrm{top}}=q_{\mathrm{top}, 0}-\left(\lambda_{o}-\lambda_{a, \mathrm{eff}}\right) \Delta T_{\mathrm{ML}} \equiv q_{\mathrm{top}, 0}-\lambda \Delta T_{\mathrm{ML}} .
$$

We of course expect the feedback $\lambda$ to be positive, since the oceans lose energy when they are warmer.

Below the mixed layer, we have eddy diffusion. We assume a fixed eddy diffusion coefficient $\kappa$, such that the temperature diffuses according to:

$$
\frac{\partial \Delta T}{\partial t}=\kappa \frac{\partial^{2} \Delta T}{\partial z^{2}}
$$

Here we implicity neglect the effect of upwelling, which is expected to be unimportant over the 11-year solar cycle, since the latter is much shorter than the typical time scale it takes to diffuse down to the thermocline at $H_{\text {therm }} \sim 400$ m (e.g., Lindzen and Giannitsis [1998]). That is, upwelling is expected to be important on times scales of order $H_{\text {therm }}^{2} / \kappa \sim 50 \mathrm{yr}$, or longer.

For simplicity, we define $z=0$ to be at the boundary between the ML and the deep ocean. From continuity, we have $\Delta T_{M L}=\Delta T(z=0)$.

The last boundary condition is on the flux between the ML and the deep ocean:

$$
q_{\mathrm{bottom}}=-\left.\kappa \frac{\partial \Delta T}{\partial z}\right|_{z=0} .
$$

Next, we assume a harmonic form for our variables. In particular, the temperature in the deep ocean is of the form $\Delta T(z, t)=\tilde{T} \exp (i k z-i \omega t)$. Note that we use squiggle marks to denote the harmonic amplitude of the different variables, as opposed to the actual time dependent values. Thus, the diffusion equation (eq. 4) gives:

$$
-i \omega \tilde{T}=-\kappa k^{2} \tilde{T} \Rightarrow k=\sqrt{\frac{\omega}{2 \kappa}}(1+i) .
$$

Once we plug the boundary conditions (eqs. 3,5) in the energy conservation equation (eq. 1), we find

$$
-i \omega \ell C_{p} \tilde{T}=\tilde{q}_{t o p, 0}-\lambda \tilde{T}+i \kappa k \tilde{T}
$$

or,

$$
\tilde{T}=\frac{\tilde{q}_{t o p, 0} / C_{p}}{\left(\sqrt{\frac{\omega \kappa}{2}}+\frac{\lambda}{C_{p}}\right)-i\left(\omega \ell+\sqrt{\frac{\omega \kappa}{2}}\right)} .
$$
is:

The relation between the absolute value of the amplitudes

$$
|\tilde{T}|=\frac{\left|\tilde{q}_{t o p, 0}\right| / C_{p}}{\sqrt{\left(\sqrt{\frac{\omega \kappa}{2}}+\frac{\lambda}{C_{p}}\right)^{2}+\left(\omega \ell+\sqrt{\frac{\omega \kappa}{2}}\right)^{2}}} .
$$

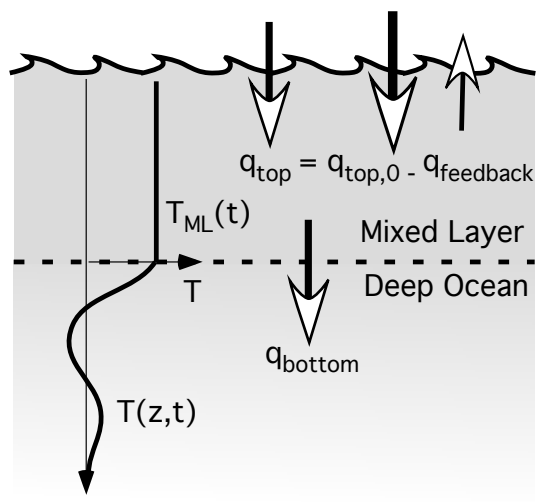

Figure 1. The 1D Ocean diffusion model we use. It is formally described by eqs. 1-5.

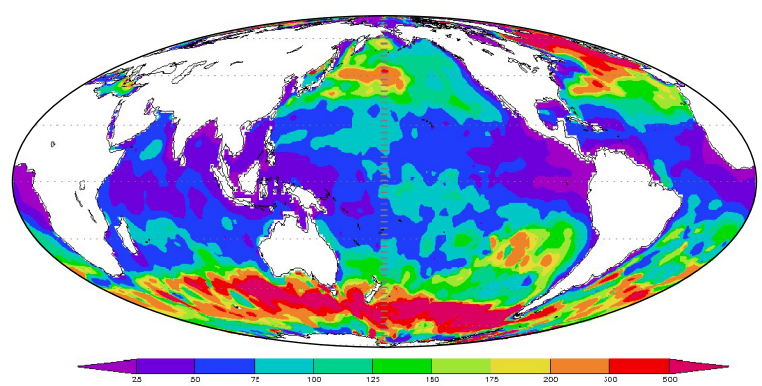

Figure 2. Maximum annual depth (in meters) of the mixed layer, based on the ocean temperature data set of Levitus and Boyer [1994].

This results provides the relation between the harmonic flux variation and the SST variations at a given point. However, we are interested in the globally averaged response.

We therefore assume for simplicity that the flux $\tilde{q}_{t o p, 0}$ is spatially constant, or at least, that it is uncorrelated with the mixing layer depth. If a correlation does exist, it would further complicate the analysis. However, as long as we do not know the spatial dependence of $\tilde{q}_{t o p, 0}$ (because we do not know the actual mechanism), this is the only reasonable assumption we can make. This allows us to define an appropriate average for an effective depth:

$$
l_{\mathrm{eff}}^{-1} \equiv\left\langle\sqrt{\left(\sqrt{\frac{\omega \kappa}{2}}+\frac{\lambda}{C_{p}}\right)^{2}+\left(\omega \ell+\sqrt{\frac{\omega \kappa}{2}}\right)^{2}}\right\rangle^{-1}
$$

where the average is taken over the global ocean area. This effective depth can then be used to relate between the flux and average temperature response:

$$
\langle\tilde{T}\rangle=\frac{\tilde{q}_{t o p, 0}}{C_{p} \ell_{\mathrm{eff}}}
$$

Note that $\ell_{\text {eff }}$ is the effective mixed layer depth that would be required, if there was no deep ocean, and no atmospheric feedbacks, to give the correct relation between the mixed layer temperature and the flux going into it.

The phase $\varphi$ between the flux going into the ocean and the surface temperature can also be derived:

$$
\tan \varphi=\frac{\sqrt{\omega \kappa / 2}+\omega \ell}{\sqrt{\omega \kappa / 2}+\lambda / C_{p}} .
$$




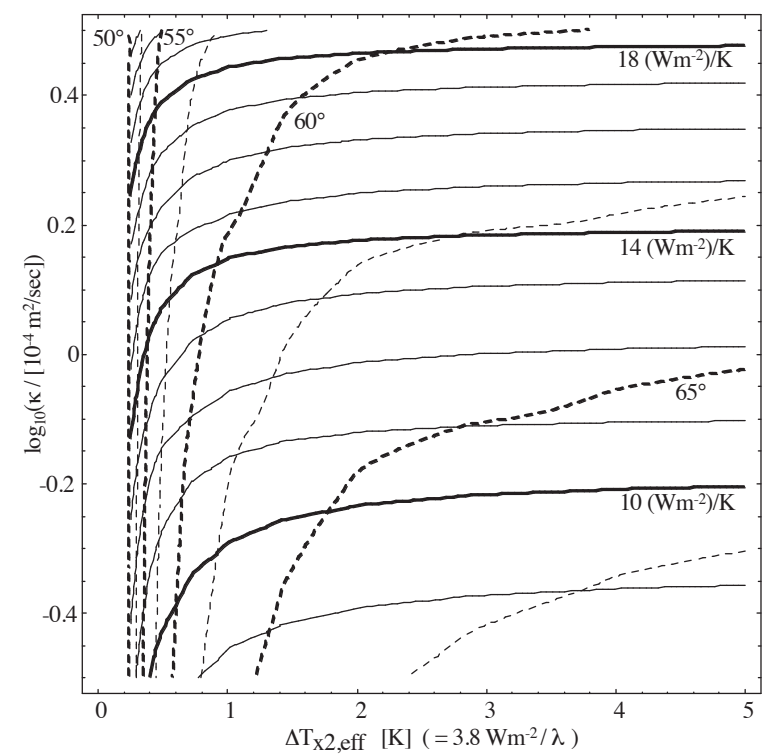

Figure 3. The model based ratio $\left|q_{\mathrm{top}, 0} / T_{\mathrm{ML}}\right|$ between the flux going into the oceans $q_{\mathrm{top}, 0}$ and the mixed layer (or sea surface) temperature $T_{\mathrm{ML}}$ (in solid contours), and the temperature phase lag behind the flux (in dashed contours), as a function of the eddy diffusivity below the mixed layer and the feedback parameter $\lambda$ as given by $\Delta T_{\times 2}=(3.8$ $\left.\mathrm{W} / \mathrm{m}^{2}\right) / \lambda$. The latter is the ocean surface temperature increased following a doubled $\mathrm{CO}_{2}$ level (which is similar to the standard used definition of sensitivity). The observed phase lag is $30^{\circ}$ to $50^{\circ}$ [White et al., 1997], thus favoring a large $\lambda$ or a low effective temperature sensitivity.

We see that if the mixed layer is large, the phase lag approaches $90^{\circ}$. If the diffusion into the deep ocean is dominant, the preferred phase is $45^{\circ}$, while the lag will tend to disappear if $\lambda$ is large (climate sensitivity is small).

The frequency we use is of course that of the 11 year solar cycle: $\omega=2 \pi / 11 \mathrm{yr}$.

Values for the diffusion coefficient were obtained in the literature using direct diffusion measurements. They range from $2 \times 10^{-5} \mathrm{~m}^{2} / \mathrm{sec}$ to $3 \times 10^{-4} \mathrm{~m}^{2} / \mathrm{sec}$, as can be seen, for example, in fig. 13 of Law et al. [2003]. Thus, we take as a nominal value $\kappa \sim 10^{-4} \mathrm{~m}^{2} / \mathrm{sec}$. Interestingly, it is also the typical value which fits the absorption of bomb ${ }^{14} \mathrm{C}$ into the oceans [Siegenthaler and Joos, 1992].

The feedback parameter $\lambda$, which is the inverse of the SST sensitivity to changes in the energy budget, is expected to be similar to the inverse of the global temperature sensitivity. The latter is often expressed as the equilibrium temperature rise expected following the doubling of the atmospheric $\mathrm{CO}_{2}$, which is equivalent to a radiative forcing of $3.8 \mathrm{~W} / \mathrm{m}^{2}$. For a gray body earth without any feedbacks, this temperature rise is $\Delta T_{\times 2}=1.2^{\circ} \mathrm{C}$. According to the IPCC-AR4, it is likely to be higher duo to strong positive feedbacks, that is, $\Delta T_{\times 2}=2-4.5^{\circ} \mathrm{C}$. Thus, we expect $\lambda \sim 3.8 \mathrm{~W} / \mathrm{m}^{2} / \Delta T_{\times 2}$ with the large aforementioned range for $\Delta T_{\times 2}$. Because the global sensitivity is still unknown, we leave $\lambda$ as a free parameter.

Last, we require the global distribution of the maximum annual mixed layer depths, which is derived from the data set of Levitus and Boyer [1994]. The distribution of maximum annual ML depths, which is depicted in fig. 2, is then used to calculate the average of eq. 10 for given diffusivities and sensitivities.

With the effective mixed layer depth calculated, we can obtain $q_{\mathrm{top}, 0} /\langle\tilde{T}\rangle$ and the phase lag. These are depicted in fig. 3 .

From fig. 3 it is apparent that the main uncertainty in determining the relation between the ocean flux and the SST is the diffusion coefficient beneath the mixed layer. For the aforementioned ranges of diffusivities, we find

$$
\left|\frac{\tilde{q}_{t o p, 0}}{T_{S S T}}\right|=13 \pm 4 \frac{W / m^{2}}{{ }^{\circ} \mathrm{C}} .
$$

\subsection{Empirical relation between the OHC and SLR}

Over the decadal time scale, thermal expansion appears to be the main contribution towards sea level rise (e.g., $§ 5.5 .6$ in the IPCC AR4). Specifically, it appears to constitute about $\left.(d h / d t)\right|_{t h} /\left.(d h / d t)\right|_{\text {tot }}=0.57 \pm 0.13$ of the total rise observed between 1993 and 2003 (and about $0.38 \pm 0.06$ on the longer, 1955 to 2003 period, less relevant to us). This implies that to a large extent SLR reflects the heat content variations, at least on the 11-year time scales, since it is comparable to all the other contributions combined.

Besides the fact that SLR are not all due to thermal expansion, we also need to consider that the actual expansion depends on the temperature and pressure. Thus, the relation between the thermal-SLR change $d h /\left.d t\right|_{t h}$ and the oceanic flux $q_{t o p}$ is not necessarily one to one. To estimate the thermal-SLR to oceanic flux ratio, we can look at the thermal SLR calculated from the observed OHC variations. We do so with both Ishii et al. [2006] and Levitus and Boyer [1994] data, and compare it to the actual OCH variations. The ratio found is

$$
\frac{\left|\tilde{q}_{t o p}\right|}{d h /\left.d t\right|_{t h}}=0.51 \pm 0.1 \frac{\mathrm{W} / \mathrm{m}^{2}}{\mathrm{~mm} / \mathrm{yr}},
$$

As a consistency check, we can also estimate this number under the crude approximation that the oceans heat uniformly, and that the expansion coefficient $\lambda$ is linear in the temperature. Under this approximation, the the ratio is

$$
\frac{\left|\tilde{q}_{t o p}\right|}{d h /\left.d t\right|_{t h}} \approx \frac{\left.C_{p}\right|_{T_{a v r}}}{\lambda} \approx 0.74 \frac{\mathrm{W} / \mathrm{m}^{2}}{\mathrm{~mm} / \mathrm{yr}},
$$

where we have taken the average ocean surface temperature $T_{a v r}$ to be $17^{\circ} \mathrm{C} . C_{p}$ is the heat capacity per unit volume (at constant pressure) of sea water. The small inconsistency implies that warmer oceans tend to heat or cool more than colder oceans, which have a smaller expansion coefficient.

Thus, to obtain the oceanic flux from the observed (total) SLR, we use the above numbers:

$$
\frac{\left|\tilde{q}_{t o p}\right|}{d h /\left.d t\right|_{t o t}}=\frac{\left|\tilde{q}_{t o p}\right|}{d h /\left.d t\right|_{t h}} \frac{d h /\left.d t\right|_{t h}}{d h /\left.d t\right|_{t o t}}=0.29 \pm 0.09 \frac{\mathrm{W} / \mathrm{m}^{2}}{\mathrm{~mm} / \mathrm{yr}} .
$$

\section{Deriving the Oceanic Heat Flux}

\subsection{Heat Flux from the Ocean Heat Content}

We begin with a direct reconstruction of the global oceanic heat flux using the thermal heat content of the top $700 \mathrm{~m}$ of the global oceans over the past 5 decades [Ishii et al., 2006], and differentiate it. Since we do not expect the 11-year oscillations to penetrate deeper than about $200 \mathrm{~m}$ (depending on the mixed layer depth and the actual diffusion coefficient), there is no need to add deeper ocean data, which would just add noise.

Two sets are generated, one for the whole ocean and one for the Atlantic region (all waters between $80^{\circ} \mathrm{W}$ and $30^{\circ} \mathrm{E}$ ). The data is then differetiated to get a flux, and averaged with a 3 year (central) running average. The result is depicted in fig. 4. The flux is the average flux going into the oceans. This is not necessarily the average over the whole globe, as it depends on the actual flux variations over land. This point is elaborated in $\S 5$. 
Evidently, there are some variations which resemble the solar activity changes, either as measured directly with the TSI [Lean, 2000], or through the CRF proxy (specifically, the Huancayo/Haleakala low geomagnetic latitude neutron monitor). The Pearson correlation coefficient is $r=-0.29$ with the CRF (negative, since more cosmic rays imply a less active sun), or 0.24 with the TSI.

$N_{\text {eff }}$, the effective number of d.o.f, is estimated in this and other analyses here using the standard Bartlett's formula [Bartlett, 1935] which implicitly assumes that the random realizations of the null hypothesis (of no correlation) signals we wish to rule out have the same autocorrelation function as those of the signals, which in our case have some periodicity. Since the general null hypothesis signals could be more general than the quasi-periodic signals we have, $N_{\text {eff }}$ is somewhat underestimated. Here we find that the number of d.o.f is 35 for the correlation with the CRF and 32 for the correlation with the TSI, giving a statistical significance of $p=0.04$ or $p=0.1$ respectively, assuming a two-tailed distribution.

One of the reasons for the poor correlation is a significant contribution from other sources. For example, it was suggested that the El-Nino southern oscillation (ENSO) which dominates the Pacific basin [Lombard et al., 2005], and volcanoes [AchutaRao et al., 2007] will give rise to large contributions to the heat flux. Another problem is that of completeness-large regions lack proper coverage [AchutaRao et al., 2007], which can give rise to spurious variability. Thus, we repeat the analysis as before, but constrain it to the Atlantic ocean (i.e., all waters between $80^{\circ} \mathrm{W}$ and $30^{\circ} \mathrm{E}$ ). The result, depicted in fig. 4, exhibits a higher correlation with solar activity variations, though some discrepancies still exist. Here $r=-0.47$ with $N_{\text {eff }}=27$ for the correlation with the CRF, and $r=0.45$ and $N_{\text {eff }}=23$ for the correlation with the TSI, giving $p=0.01$ and 0.02 respectively.



Figure 4. Sea Surface Temperature anomaly, Sea Level Rate, Net Oceanic Heat Flux, the TSI anomaly and Cosmic Ray flux variations. In the top panel are the inverted Haleakala/Huancayo neutron monitor data (heavy line, dominated by cosmic rays with a primary rigidity cutoff of $12.9 \mathrm{GeV}$ ), and the TSI anomaly (TSI $-1366 \mathrm{~W} / \mathrm{m}^{2}$, thin line, and based on Lean [2000]). The next panel depicts the net oceanic heat flux, averaged over all the oceans (thin line) and the average heat flux in the Atlantic region (Lon $80^{\circ} \mathrm{W}$ to $30^{\circ} \mathrm{E}$, thick line), based on Ishii et al. [2006]. The next two panels plot the SLR and SST anomaly. The thin lines are the two variables with their linear trends removed. In the thick lines, the ENSO component is removed as well (such that the cross-correlation with the ENSO signal will vanish).
Since the correlation with the TSI and with the CRF are not meaningfully different, this correlation cannot be used to say, at face value, whether the large solar effect is directly related to solar activity (and the TSI), or indirectly, through modulation of the cosmic ray flux. Hence, we can conclude that a large solar influence exists, we can quantify it, but we cannot determine the physical origin of the influence from just the correlation. Note also that the best fit lags have rather large errors, of at least 6 months. If we also consider that the phase within the solar cycle cannot be as accurately defined, we can deduce that the lags do not lead to any meaningful conclusions.

Another way of visualizing the results, it to fold the data over the 11-year solar cycle and average. This reduces the relative contribution of sources uncorrelated with the solar activity as they will tend to average out (whether they are real or noise). The results for the $\mathrm{OHC}$ can be seen in fig. 5. Here the correlation between the TSI and $\mathrm{OHC}$ is about $r=0.8$ for both the Atlantic and Global OHC data. Although the statistical significance of this much higher correlation is similar to the above analysis of the unfolded data, the folded analysis visually picks out the solar-signal within the data. A $\chi^{2}$ fitting of the somewhat higher quality Atlantic $\mathrm{OHC}$ variations gives that:

$$
\left.\frac{\left|\tilde{q}_{t o p}\right|}{\Delta(T S I)}\right|_{O H C}=1.05 \pm 0.25 \text {. }
$$

Note that the number is dimensionless.



Figure 5. From top to bottom: The sea surface temperature anomaly, the Global $\mathrm{OHC}$ variations and the Atlantic OHC variations from fig. 4 folded over the solar cycle and averaged. Two cycles (with solar minimum being $\phi=0)$ are shown. The solid lines are $\chi^{2}$ fits to a harmonic variation. Unlike fig. 4 , the $\mathrm{OHC}$ data is not box averaged with a 3 year running average. Instead, it is averaged into 1-year bins then pairwise differentiated. This implies that nearby points in the $\mathrm{OHC}$ variations are correlated but significantly less than in fig. 4 . The SST data is yearly averaged, but since there is no derivative, the points are uncorrelated. The figure demonstrates that once the data is folded over the solar cycle, the uncorrelated noise is suppressed. This leaves a much clearer correlation. In fact, the correlation coefficients between the SST, Global and Atlantic OHC variations, and the reconstructed solar flux are $r=0.83,0.79,0.86$ respectively. Note that the statistical significance of the result is not markedly different than in the case of the unfolded data because the effective number of degrees of freedom is much smaller here. 


\subsection{Heat flux from the Sea Level Change Rate}

Given the relatively small correlation coefficient and modest significance, it is worth while to corroborate the existence of the large heat flux variations using an independent data set. We thus turn to analyze tide gauge data measuring sea level variations. Note however that this dataset by itself cannot be used to quantify the total oceanic heat content without knowing the actual temperature distribution and its variations, since the thermal expansion coefficient is temperature dependent. Nonetheless, if heat content variations do take place, they give rise to a changed sea level. This implies that we can independently check that the oscillations in the heat content are indeed related to solar variability, because if they are, the tidal data should too exhibit oscillations, but extending over the longer time span of the data.

The tide based sea level change data set we construct uses 24 stations previously chosen by Douglas [1997] to satisfy several constraints: (1) Long record (at least 60 years), (2) not located near collisional plate boundaries, (3) at least $80 \%$ complete, (4) show reasonable agreement with nearby gauges, and (5), not located in regions subject to large post-glacial rebound. The data sets themselves were obtained from the Permanent Service for Mean Sea Level (http://www.pol.ac.uk/psmsl). To obtain a continuous data set, the data from the different stations must be averaged and differentiated. If this procedure is carried out at this order, then the differentiation may introduce spurious noise between yearly time bins where stations are added or removed. Thus, the station data is first pairwise differentiated, that is, the sea level change between each two consecutive years is first computed for each station separately. Then, the mean of the annual sea level change rates is calculated. Although this order avoids spurious derivatives, it

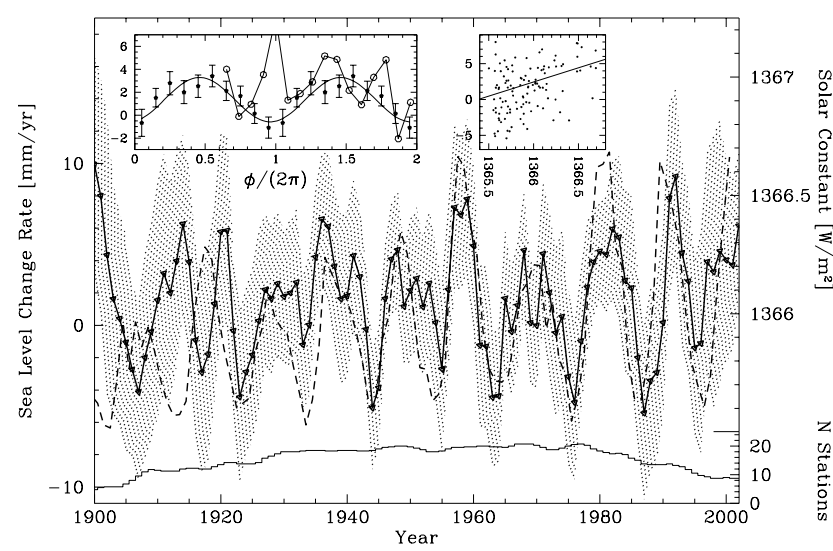

Figure 6. Sea Level vs. Solar Activity. Sea level change rate over the $20^{\text {th }}$ century is based on 24 tide gauges previously chosen by Douglas [1997] for the stringent criteria they satisfy (solid line, with $1-\sigma$ error range denoted with the shaded region). The rates are compared with the total solar irradiance variations Lean [2000] (dashed line, with the secular trends removed). Here $r=0.54$ giving a $p=10^{-4}$ (for $N_{\text {eff }}=47$ ). The inset depicts the sea level change rate folded over the solar cycle together with a sinusoidal least $\chi^{2}$ fit (each year is assigned a phase relative to the preceding and following solar minima, after which all data points within a phase bin are averaged; the data is then shown twice, over two cycles, for clarity). The tide gauge data leads the solar forcing by $3 \pm 6$ months. The Inset also depicts the TOPEX / Jason satellite based sea level change rate overlaid on the solar-cycle folded tide-gauge data. Besides the large 1997 El-Niño event, the two different data sets are consistent with each other. can produce long term drifts once the data set is integrated. This spurious effect, however, is not relevant for the present analysis in which only the 11-year cycle is important. The data is subsequently averaged with a 3 -year (central) running mean. This removes some of the short time scale noise without compromising the 11-year average.

The reconstructed sea level variation rate is portrayed in fig. 6. A very clear correlation between solar activity and sea level is evident. The correlation deteriorates when going back to before about 1920, which is not unexpected given the small number of stations (also manifested in the larger estimated error range) and the poor coverage of the Pacific. Note also that these oscillations are also consistent with previous analyses. In particular, Holgate and Woodworth [2004], which is based on a tide gauge record constructed using 177 stations between 1953 to 2003, exhibits the same 11 year periodicity and phase.

Here we find a correlation coefficient of $r=0.55$ with the solar luminosity reconstruction [Lean, 2000]. Unlike the previous record, the correlation extends over many more solar cycles. The high $N_{\text {eff }}=67$, gives rise to a $99.99 \%$ confidence that random realizations with similar autocorrelation functions as the actual signals cannot give such a high coefficient $r$.

A linear regression between the SLR and TSI gives a slope of $5.8 \pm 0.5 \mathrm{~mm} / \mathrm{yr}$ per $\mathrm{W} / \mathrm{m}^{2}$. Using the results of eq. 16 , this implies that

$$
\left.\frac{\left|\tilde{q}_{t o p}\right|}{\Delta(T S I)}\right|_{S S T}=1.68 \pm 0.6 .
$$

The largest contribution to the error is the uncertainty between the thermal and total sea level variations over the 11-year time scale.

As a consistency check, it is interesting to compare the tide-gauge record with satellite data. We therefore take the combined TOPEX / Jason sea level data sets [Beckley et al., 2007, see also http://sealevel.colorado.edu/]. We average it set over 1-year bins, and then pairwise differentiate it. The result is plotted in fig. 6 , overlaid on the folded tide-gauge based SLR. The large discrepancy at solar minimum arises from the very large $1997 \mathrm{El} \mathrm{Niño} \mathrm{event.} \mathrm{Other} \mathrm{than} \mathrm{that,}$ the satellite and the folded SLR are reassuringly consistent with each other.

\subsection{Heat flux from the Sea Surface Temperature}

White et al. [1997] have shown that two different data sets, that of the bathythermograph and the Global Ice and Sea Surface Tempeature measurements, exhibit the 11-year solar cycle in the temperature. The correlation between the SST and the TSI signal found ranges between 0.08 to $0.1^{\circ} \mathrm{C} /\left(\mathrm{W} / \mathrm{m}^{2}\right)$. This is consistent with the somewhat larger values found for the correlation between temperature variations over ground and the TSI, e.g., $0.11 \pm 0.02^{\circ} \mathrm{C} /\left(\mathrm{W} / \mathrm{m}^{2}\right)$ by Douglass and Clader [2002]. Although there is no need to repeat here the analysis of White et al. [1997], the NCDC SST anomaly with the clear 11-year solar cycle signature is plotted in fig. 4.

Using eq. 13 and the $0.09 \pm 0.01{ }^{\circ} \mathrm{C} /\left(\mathrm{W} / \mathrm{m}^{2}\right)$ correlation between the SST and the TSI, we can estimate the ratio between the total ocean heat flux and theTSI to be

$$
\left.\frac{\left|\tilde{q}_{t o p, 0}\right|}{\Delta(T S I)}\right|_{S L R}=1.15 \pm 0.35 \text {. }
$$

\section{More on the data sets used}

Before deriving the global heat flux from the observed ocean heat content, it is worth while to study in more detail the different data sets we used, and in particular, to 
better understand their limitations. Since we wish to compare them to each other, we begin by creating comparable data sets, with the same resolution and time range. Thus, we down sample higher resolution data into one year bins and truncate all data sets to the range of 1955 to 2003 . We also include in this analysis the ENSO signal (using NOAA's ENSO-MEI index), since we expect it to be a major source of "noise" in the analysis.

Once constructed, all pairs of data sets are crosscorrelated, while allowing for lags between -2 and +2 years. The results are summarized in table 1 , which includes the best fit linear correlation coefficient, the lag, the effective number of degrees of freedom, calculated using the standard Bartlett formula (see $\S 3.1$ ), and the probability that the null hypothesis can be ruled out. The latter is the probability that random realization of signals with the same auto-correlation could give a correlation coefficient which is as high as observed. Note that the formal 1- $\sigma$ errors on the lags are consistently around $0.5 \mathrm{yr}$ for all fits with $r>0.3$.

Several conclusions can be drawn from the table. First, the low correlation between the global $\mathrm{OHC}$ variations and the other climatic variables (except for the Atlantic variations) strongly suggests that that the global OHC data set is significantly noisy [e.g., as suggested by AchutaRao et al., 2007]. This naturally explains why the solar related variables exhibit a lower correlation with the global OHC than with the other climatic signals.

Second, it is of course unsurprising that the ENSO has an important effect on the SST and to some extent also on the SLR. It should also come with no surprise that the Atlantic OHC does not correlate with the ENSO index. The fact that there is no correlation with the global OHC can be attributed to two effects. First, because time scales associated with the ENSO are relatively fast, the heat variations do not penetrate deep into the ocean. This implies that the ENSO could be important for the SST variations but not the $\mathrm{OHC}$, which includes a large oceanic volume, down to $700 \mathrm{~m}$ in depth. And of course, the global $\mathrm{OHC}$ has a significant amount of noise.

One can remove the ENSO component from the SST and the SLR, the result of which is depicted in fig. 4. Although the SLR does not change much, the effect on the SST is to remove much of the faster oscillations, leaving a signal with a clear signature of the 11-year solar cycle.

Last, because there is no statistically significant difference between correlations with the total solar irradiance and with the cosmic ray flux, it is not possible with this data to point out whether a mechanism directly related to solar activity, or an indirect mechanism related to CRF variations is responsible for the large heat fluxes.

\section{Oceanic flux vs. global radiative forcing}

The next step is to estimate the total flux into the oceans $q_{t o p, 0}$, derive the average global radiative forcing, and then compare it with different expectations for the forcing variations.

If the oceans were hypothetical reservoirs with an infinite heat capacity, all the heat going into them would remain, and it would not be reemitted from the surface. In such a case, the observed heat content variations would reflect the real variations in the short wavelength radiation going into the oceans, that is, $q_{t o p, 0}=q_{t o p}$. In reality, however, the finite heat capacity of the oceans implies that as heat is absorbed, the temperature increases. Once the surface temperature changes, it would begin emitting or absorbing heat (as long wavelength radiation, latent heat of evaporation, etc). This loss of heat implies that the calorimetric efficiency is less than unity.

In terms of eq. 3, the ocean heat content hitherto measured with the OHC and SLR was $q_{t o p}$, whereas we are interested in $q_{t o p, 0}$. Assuming we know $\lambda$ and $\Delta T_{M L}$, the amplitude of total flux variations is therefore

$$
\left|\tilde{q}_{t o p, 0}\right|=\sqrt{\left|\tilde{q}_{t o p}\right|^{2}-\left(\lambda \Delta T_{M L} \sin (\theta)\right)^{2}}+\lambda \Delta T_{M L} \cos (\varphi)
$$

here $\theta$ is the phase lag between the ML temperature signal and the solar signal, i.e., $q_{t o p, 0}$ (not the observed heat flux $\left.q_{\text {top }}\right)$. It is observed to be $30^{\circ}$ to $50^{\circ}$ [White et al., 1997]. The same authors also found that the surface temperature varies with a peak to peak amplitude of 0.08 to $0.10 \mathrm{~K}$ over the solar cycle. Thus, the largest uncertainty in determining the total flux going into the oceans is by far the relatively unknown sensitivity $\lambda$.

As described after eq. 20, we expect the ocean sensitivity to be similar, or perhaps somewhat smaller than the global temperature sensitivity. The sensitivity itself is often parameterized as $\lambda \sim 3.8 \mathrm{~W} / \mathrm{m}^{2} / \Delta T_{\times 2}$, with $\Delta T_{\times 2}$ being the $\mathrm{CO}_{2}$ doubling temperature sensitivity. According to the IPCC-AR4, the global temperature sensitivity is most likely in the range $\Delta T_{\times 2}=2-4.5^{\circ} \mathrm{C}$. Since the oceanic sensitivity may be somewhat smaller, and because some evidence suggests [e.g., Shaviv, 2005] that the global climate sensitivity is on the low side, we will conservatively take the range of $\Delta T_{\times 2}=1-5^{\circ} \mathrm{C}$, for the calculation of $\lambda$ and $\tilde{q}_{t o p, 0}$.

For example, if we consider the directly measured heat content variations (eq. 17), then we find that

$$
\left.\frac{\left|\tilde{q}_{t o t, 0}\right|}{\Delta(T S I)}\right|_{O H C}=1.2 \pm 0.3 \text {. }
$$

Note that because the measured $\tilde{q}_{t o p}$ is typically $1 \mathrm{~W} / \mathrm{m}^{2}$ or larger, but the $\lambda \Delta T_{M L}$ is at most about $0.35 \mathrm{~W} / \mathrm{m}^{2}$, the outgoing flux from the ocean is at most a modest correction. It also implies that the calorimetric efficiency is relatively high, typically between 80 to $95 \%$.

Another point to consider is the fact that the flux obtained from eq. 21 is only the flux above the oceans. Two extreme limits can then follow. If the mechanism responsible for the flux variations operates only over the oceans, than the globally averaged flux $F_{\text {global }}$ will be $70 \%$ of the oceanic flux, that is, $F_{\text {global }} \approx 0.7\left|\tilde{q}_{t o p, 0}\right|$. In the opposite limit, where the mechanism operates equally above land and ocean, but none of the land flux ends up in the oceans, the globally averaged flux will be $F_{\text {global }} \approx\left|\tilde{q}_{t o p, 0}\right|$. Thus,

$$
\left.\frac{F_{\text {global }}}{\Delta(T S I)}\right|_{O H C}= \begin{cases}0.85 \pm 0.2 & F \text { only over oceans } \\ 1.2 \pm 0.3 & \text { over land and ocean }\end{cases}
$$

The results for the ocean heat content indirectly derived from the SLR and SST are summarized in fig. 7. We will adopt eq. 22 as out best estimate for the global radiative forcing variations. In principle, we could also factor in the results from the SLR and SST analysis and thus reduce the statistical error for the best estimate. We will not do so however, because the $\mathrm{OHC}$ data provides a much more direct measurement of the radiative forcing, one which is less prone to the effects of systematic errors. In particular, the SLR data includes volume changes not associated with thermal expansion, or the SST based measurement depends on theoretical modeling with a few poorly determined parameters. Examples for the latter include the climate sensitivity, heat diffusion coefficient or the fact that we implicitly assume that the mixed layer depth is not correlated with the spacial dependence of the radiative forcing. In other words, we use the SLR and SST for two primary reasons. The data sets prove with a high statistical probability that the solar cycle does manifest itself in the data, and they also provide independent consistency checks for the observed magnitude 
Table 1. Cross-correlation between the different solar and climatic signals. Higher resolution data is down sampled to 1 year bins, and all data is truncated to the range between 1955 - 2003 so as to be comparable with each other. The upper right half summaries the correlation coefficient $r$ (big) and the lag in years of the best fit (small, positive number implies that the column variable lags behind the row variable). The bottom left numbers summarize the effective number of d.o.f. in the cross-correlation (using Bartlett's formula, 3 should be subtracted due to the fit), and the statistical significance of the correlation coefficient (the probability that random realizations with the same number of degrees of freedom could yield a value of $r$ as least has high as the correlation). The CRF is the Huancayo/Haleakala data, hence we use $-\Phi_{C R F}$. The OHC's are the time derivatives of the heat content. Note that between the Global OHC derivative and the ENSO there is no positive correlation for any lag between -2 to +2 years, hence the " $x$ ".

\begin{tabular}{cccccccc}
\hline & $L \odot$ & $-\Phi_{C R F}$ & Gbl. OHC & Atl. OHC & SLR & SST & ENSO \\
\hline$L_{\odot}$ & - & $0.84,0.8$ & $0.18,-0.2$ & $0.41,1.3$ & $0.72,0.6$ & $0.42,0.2$ & $0.15,1.5$ \\
$-\Phi_{C R F}$ & $30,0.0006 \%$ & - & $0.25,-0.6$ & $0.42,0.5$ & $0.61,-0.2$ & $0.48,-0.5$ & $0.17,0.9$ \\
Gbl. OHC & $27,19 \%$ & $26,12 \%$ & - & $0.73,0.5$ & $0.11,0.1$ & $0.21,0.1$ & $\times$ \\
Atl. OHC & $26,2.4 \%$ & $26,2 \%$ & $26,0.02 \%$ & - & $0.37,-0.5$ & $0.24,-0.5$ & $0.08,-0.7$ \\
SLR & $24,0.05 \%$ & $26,0.2 \%$ & $29,29 \%$ & $28,3 \%$ & - & $0.32,-0.6$ & $0.37,0.4$ \\
SST & $35,0.8 \%$ & $36,0.3 \%$ & $33,13 \%$ & $36,8.4 \%$ & $38,3 \%$ & - & $0.52,-0.7$ \\
ENSO & $43,17 \%$ & $44,14 \%$ & $\times$ & $45,30 \%$ & $45,0.8 \%$ & $42,0.05 \%$ & - \\
\hline
\end{tabular}

of the ocean heat content variations. But it is hazardous to use them for determining the exact value of the global radiative forcing.

Note that the relatively low correlation coefficient between the $\mathrm{OHC}$ and the solar signals may seem somewhat suspicious. Nevertheless, the relatively large number of degrees of freedom implies that the Atlantic OHC does have a significant correlation, at the 0.01 to 0.02 level with the different solar variables. Moreover, the solar cycle folded OHC data revealed (in fig. 5) that even the noisier global $\mathrm{OHC}$ does exhibit a clear solar-cycle periodicity in it. Namely, the OHC data suffers from large non-solar contributions (whether noise or real signals), but it does exhibit large solar-cycle variations as well.

\section{Discussion}

The present work clearly demonstrates that there are large variations in the oceanic heat content together with the 11-year solar cycle. Three independent data sets consistently show that the oceans absorb and emit an order of magnitude more heat than could be expected from just the variations in the total solar irradiance.

Grossly speaking, there are three alternative explanations to the large flux. As we shall see, only the first is in fact viable:

1. The straight forward explanation to the well detected and documented heat content variations is that the large amount of heat periodically entering the oceans simply reflect real variations in the energy budget, a flux which is not part of any internal feedback mechanism, whether oceanic or atmospheric. For example, if the amount of cloud cover is directly influenced by solar activity (irrespective of any atmospheric feedback in which they play), it would serve as an iris which periodically allows large amounts of heat to enter or leave the climate system. That is, it is an externally controlled "valve".

2. Alternatively, one can imagine that the flux is not "external" but a result of internal feedbacks in the climate system. Namely, the TSI variations are the source of a small fraction of the flux entering the oceans, while the rest of the flux arises from a coupling between the sea surface temperature and the climate system. For example, a higher sea surface temperature is responsible for more water vapor in the atmosphere, which as a greenhouse gas, re-radiates heat back into the oceans, giving rise to a strong positive feedback.

Numerically, we require a feedback flux of order $1 \mathrm{~W} / \mathrm{m}^{2}$, from the observed SST variations of $\sim 0.1^{\circ} \mathrm{C}$, or a feedback parameter of $\lambda \sim 10\left(\mathrm{~W} / \mathrm{m}^{2}\right) /{ }^{\circ} \mathrm{C}$. However, all the known feedbacks, with all their uncertainties are typically between -1 to $2\left(\mathrm{~W} / \mathrm{m}^{2}\right) /{ }^{\circ} \mathrm{C}$ in equilibrium [e.g., Soden and Held, 2006]. Namely, they are about an order of magnitude too small to explain the heat flux.

Moreover, not only is there no known feedback, it is incompatible with the theoretical results of $\S 2.1$. A large atmospheric feedback is equivalent to a large $\lambda_{a \text {,eff }}$. However, there is an upper limit on $\lambda_{a \text {,eff }}$. As it approaches $\lambda_{o} \sim 3\left(\mathrm{~W} / \mathrm{m}^{2}\right) /{ }^{\circ} \mathrm{C}$ (for the gray body ocean), the value of $\lambda$ will vanish, implying that the climate sensitivity diverges. A negative $\lambda$ gives rise to a climate system that is unconditionally unstable. In other words, the large required feedback will simply make the climate system unstable, which it is not.

Another way of looking at it, is with fig. 3. For any possible diffusivity $\kappa$ or any sensitivity $\lambda$, there is a lower limit of about $8\left(\mathrm{~W} / \mathrm{m}^{2}\right) /{ }^{\circ} \mathrm{C}$ to the ratio between $q_{t o p, 0}$ and the SST variation. This means that there is no atmospheric feedback operating on the SST which can explain the SST variations of $0.1^{\circ} \mathrm{C}$ from the meagre $0.17 \mathrm{~W} / \mathrm{m}^{2}$ variations in the TSI.

3. The third possibility is that the apparently large amounts of energy entering the oceans are actually fictitious. This could arise if the the small TSI variations excite a decadal oscillation mode. That is, a mode which contains 10 times as much energy as supplied by the TSI, is excited, such that the thermal component of the mode would appear to vary by a factor much larger than the energy supplied. This will naturally arise if an oscillator with a large $\mathrm{Q}$ value is excited near its resonance. Note that oceanic oscillation modes are known to exist. For example, modes involving coupled Rossby and Kelvin waves can beautifully explain different aspects of the ENSO for example [Graham and White, 1988]. Here we can think of two sub-cases:

(i) In the first case, we would expect the energy contained in the mode to oscillate between the observed thermal content and another type of energy contained in the oceans, where the damping of the mode is replenished by the small TSI variations. This would make the heat content appear to have large variations, with only a small energy being supplied. This interpretation, however, can be easily ruled out. The reason is that there is no other form of energy that can participate in the oscillation and which would have stayed undetected, since only the top $100 \mathrm{~m}$ appear to participate in the mode [White et al., 2000]. For example, the few $\times 10^{22} \mathrm{~J}$ would require velocities of several $\mathrm{m} / \mathrm{s}$ if the energy is kinetic or height variations of several meters if the energy is gravitational. In other words, the observed flux into the oceans is real-all this energy must enter and leave the oceans every cycle. 
(ii) The second case is to have the small TSI variations excite an oceanic mode which couples to the atmosphere above and periodically lets a variable heat flux enter the oceans. Here the heat flux is real, but most of it does not originate with the TSI variations. Instead, it arises from a positive atmospheric feedback response that is coupled to the oceanic oscillation, which allows more radiation be absorbed while the temperature is higher. This is similar to the second possibility above, except that we assume specifically that the feedback is coupled to the decadal mode, and will not be present under equilibrium. This will avoid the limitation of a negative feedback $\lambda$ and ensuing instability. Namely, the climate system could be unstable to a decadal like mode, i.e., one which self excites, but which is tuned to the external forcing of the TSI.

There are three main problems with this interpretation. First, the observed phases are inconsistent. The heat flux and sea level change rate appear to be synchronized with the solar activity variations (e.g., fig. 6). On the other hand, any atmospheric response would be insensitive to the flux getting into the oceans, and instead depend on the SST which appears [White et al., 1997] to lag behind the solar activity by $30^{\circ}$ to $50^{\circ}$ (as one would expect from the diffusion into a semi-infinite medium, which predicts $45^{\circ}$ ). An additional lag of typically $90^{\circ}$ should be expected given that we are



Figure 7. Summary of the "calorimetric" measurements and expectations for the average global radiative forcing $F_{\text {global }}$. Each of the 3 measurements suffers from different limitations. The ocean heat content $(\mathrm{OHC})$ is the most direct measurement but it suffers from completeness and noise in the data. The heat flux obtained from the sea surface temperature (SST) variations depends on the modeling of the heat diffusion into the ocean, here the diffusion coefficient is the main source of error. As for the sea level based flux, the largest uncertainty is due to the ratio between the thermal contribution and the total sea level variations. The solid error bars are the global radiative forcing obtained while assuming that similar forcing variations occur over oceans and land. The dotted error bars assume that the radiative forcing variations are only over the oceans. These measurements should be compared with two different expectations. The TSI is the expected flux if solar variability manifests itself only as a variable solar constant. The "Low Clouds+TSI" point is the expected oceanic flux based on the observed low altitude cloud cover variations, which appear to vary in sync with the solar cycle (while assuming several approximations). Evidently, the TSI cannot explain the observed flux going into the ocean. An amplification mechanism, such as that of CRF modulation of the low altitude cloud cover is required. exciting a mode near its resonance. Thus, we would expect in such a scenario to see the flux lag behind the forcing and the SST lag behind both, but this is not observed.

Second, irrespective of the details, any amplification through the effects of an oceanic mode would tend to give SST variations which are different in different basins (e.g., the eigenmode of the ENSO is primarily confined to the Pacific). However, the decadal oscillations appear to be quite similar in different basins, as can be seen from the heat content variations (fig. 4), or from the geographic distribution of the decadal SST variations [White et al., 1997].

Although it bares no theoretical weight, it is interesting to note that no such mode is known to exist, or to arise from numerical simulations.

We thus conclude that the apparent oceanic flux variations must be the result of a large amount of heat of an external forcing, which periodically enters and leaves the oceans without being amplified by the atmosphere nor by an internal oceanic mode. This implies that the sun affects climate through a mechanism other than TSI variations.

One possible mechanism to the large heat fluxes is that of CRF modulation [Ney, 1959; Dickinson, 1975; Svensmark, 1998]. The CRF, which inversely follows the solar activity variations, is the dominant source of tropospheric ionization. Although the idea has several pros and cons [Carslaw et al., 2002], the current supporting body of evidence is increasing steadily, though not without fierce critique. This includes correlations between CRF variations and cloud cover [Svensmark, 1998; Marsh and Svensmark, 2000b], correlations between between non-solar CRF variations and temperature over geological time scales [Shaviv, 2002, 2003; Shaviv and Veizer, 2003], as well as experimental results showing that the formation of small condensation nuclei (CNs) could be bottlenecked by the number density of atmospheric ions [Harrison and Aplin, 2001; Eichkorn et al., 2002; Svensmark et al., 2006]. It is yet to be proven, however, that the formation rate of small CNs is an important factor in determining the overall production rate of the large cloud condensation nuclei $(\mathrm{CCN})$ required for cloud condensation [Carslaw et al., 2002].

Another interesting point to note is that the solar cycle induced variations in low altitude cloud cover [Marsh and Svensmark, 2000b], presumably from CRF modulation over the oceans (where CCNs are most likely to be a bottleneck), give rise to a radiative imbalance which can be estimated [Marsh and Svensmark, 2000a; Shaviv, 2005] to be of order $1.1 \pm 0.3 \mathrm{~W} / \mathrm{m}^{2}$ over the past two cycles. Together, with the TSI variations, we find that the ratio between the cloud+TSI variations compared with the change in the solar constant is:

$$
\left.\frac{F_{\text {clouds }}+F_{T S I}}{\Delta(T S I)}\right|_{\text {global }}=1.3 \pm 0.4 .
$$

After comparing with eq. 21, we can conclude that the heat flux going into the oceans is consistent with the apparent variations in the low altitude clouds. See also fig. 7 .

In summary, we find clear evidence indicating that the total flux entering the oceans in response to the solar cycle is about an order of magnitude larger than the globally averaged irradiance variations of $0.17 \mathrm{~W} / \mathrm{m}^{2}$. The sheer size of the heat flux, and the lack of any phase lag between the flux and the driving force further implies that it cannot be part of an atmospheric feedback and very unlikely to be part of a coupled atmosphere-ocean oscillation mode. It must therefore be the manifestation of real variations in the global radiative forcing.

It should be stressed that the observed correlation between the oceanic heat flux and solar activity does not provide proof for any particular amplification mechanism, including that of the $\mathrm{CRF} /$ climate link. It does however provide very strong support for the notion that an amplification 
mechanism exists. Given that the CRF/climate links predicts the correct radiation imbalance observed in the cloud cover variations, it is a favorable candidate.

With respect to simulating climate dynamics, the results have two very interesting ramifications. First, they imply that any attempt to explain historic temperature variations should consider that the solar forcing variations are almost an order of magnitude larger that just the TSI variations now used almost exclusively. It would imply that the climate sensitivity required to explain historic temperature variations is smaller than often concluded.

Second, an additional constraint can be used to narrow the range of GCMs' model parameters. Under solar cycle like periodic forcing, a GCM should predict that the ratio between the oceanic heat flux and sea surface temperature variations is that which is observed, namely, a net oceanic flux of $1.05 \pm 0.25 \mathrm{~W} / \mathrm{m}^{2}$ for every $0.09 \pm 0.01^{\circ} \mathrm{C}$ change in the sea surface temperature (or somewhat larger land surface temperature variations). This should prove useful in constraining GCM based predictions, such as that of climate sensitivity.

\section{References}

AchutaRao, K., M. Ishii, B. Santer, P. Gleckler, K. Taylor, T. Barnett, D. Pierce, R. Stouffer, and T. Wigley, Simulated and observed variability in ocean temperature and heat content, Proceedings of the National Academy of Sciences, 104(26), 10,768, 2007.

Bartlett, M. S., Some aspects of the time-correlation problem in regard to tests of significance, J. R. Stat. Soc., 98, 536-543, 1935.

Beckley, B. D., F. G. Lemoine, S. B. Luthcke, R. D. Ray, and N. P. Zelensky, A reassessment of global and regional mean sea level trends from TOPEX and Jason-1 altimetry based on revised reference frame and orbits, Geophys. Res. Lett., 34, 14,608, doi:10.1029/2007GL030002, 2007.

Bond, G., et al., Persistent Solar Influence on North Atlantic Climate During the Holocene, Science, 294, 2130-2136, 2001.

Carslaw, K. S., R. G. Harrison, and J. Kirkby, Cosmic Rays, Clouds, and Climate, Science, 298, 1732-1737, 2002.

Dickinson, R. E., Solar Variability and the Lower Atmosphere., Bull. Am. Met. Soc., 56, 1240-1248, 1975.

Douglas, B. C., Global Sea Rise: A Redetermination, Sur. Geophys., 18, 279-292, 1997.

Douglass, D. H., and B. D. Clader, Climate sensitivity of the Earth to solar irradiance, Geophys. Res. Lett., 29, 33-1, 2002.

Eddy, J. A., The Maunder Minimum, Science, 192, 1189-1202, 1976

Eichkorn, S., S. Wilhelm, H. Aufmhoff, K. H. Wohlfrom, and F. Arnold, Cosmic ray-induced aerosol-formation: First observational evidence from aircraft-based ion mass spectrometer measurements in the upper troposphere, Geophys. Res. Lett., 29, 43-1, 2002.

Foukal, P., C. Fröhlich, H. Spruit, and T. M. L. Wigley, Variations in solar luminosity and their effect on the Earth's climate, Nature, 443, 161-166, doi:10.1038/nature05072, 2006.

Graham, N. E., and W. B. White, The El Nino Cycle: A Natural Oscillator of the Pacific Ocean-Atmosphere System, Science, 240, 1293-1302, 1988.

Haigh, J. D., The Role of Stratospheric Ozone in Modulating the Solar Radiative Forcing of Climate, Nature, 370, 544, doi: 10.1038/370544a0, 1994.

Harrison, R. G., and K. L. Aplin, Atmospheric condensation nuclei formation and high-energy radiation, J. Atmos. Terr. Phys., 63, 1811-1819, 2001

Holgate, S. J., and P. L. Woodworth, Evidence for enhanced coastal sea level rise during the $1990 \mathrm{~s}$, Geophys. Res. Lett., 31, 7305, doi:10.1029/2004GL019626, 2004

Ishii, M., M. Kimoto, K. Sakamoto, and S. Iwasaki, Steric sea level changes estimated from historical ocean subsurface temperature and salinity analyses, J. Ocean., 62, 155-170, 2006.
Law, C., E. Abraham, A. Watson, and M. Liddicoat, Vertical eddy diffusion and nutrient supply to the surface mixed layer of the Antarctic Circumpolar Current, J. Geophys. Res, 108, 3272, 2003.

Lean, J., Evolution of the Sun's spectral irradiance since the Maunder Minimum, Geophys. Res. Lett., 27, 2425-2428, doi: 10.1029/2000GL000043, 2000 .

Levitus, S., and T. Boyer, World Ocean Atlas 1994. Volume 4. Temperature, Tech. rep., PB-95-270112/XAB; NESDIS4, National Environmental Satellite, Data, and Information Service, Washington, DC (United States) (Found at: http://iridl.ldeo.columbia.edu/SOURCES/.LEVITUS94/ ), 1994 .

Lindzen, R., and C. Giannitsis, On the climatic implications of volcanic cooling, Journal of Geophysical Research. D. Atmospheres, 103, 5929-5941, 1998.

Lombard, A., A. Cazenave, P.-Y. Le Traon, and M. Ishii, Contribution of thermal expansion to present-day sea-level change revisited, Glob. Plan. Change, 47, 1-16, 2005.

Marsh, N., and H. Svensmark, Cosmic Rays, Clouds, and Climate, Sp. Sci. Rev., 94, 215-230, 2000a.

Marsh, N. D., and H. Svensmark, Low Cloud Properties Influenced by Cosmic Rays, Phys. Rev. Lett., 85, 5004-5007, 2000b.

Neff, U., S. J. Burns, A. Mangini, M. Mudelsee, D. Fleitmann, and A. Matter, Strong coherence between solar variability and the monsoon in Oman between 9 and 6kyr ago, Nature, 411 290-293, 2001.

Ney, E. P., Cosmic radiation and weather, Nature, 183, 451, 1959.

Shaviv, N., On climate response to changes in the cosmic ray flux and radiative budget, Journal of geophysical research, 110(A 8), 8105-8105, 2005.

Shaviv, N. J., Cosmic Ray Diffusion from the Galactic Spiral Arms, Iron Meteorites, and a Possible Climatic Connection, Phys. Rev. Lett., 89(5), 051,102, 2002.

Shaviv, N. J., The spiral structure of the Milky Way, cosmic rays, and ice age epochs on Earth, New Astron., 8, 39-77, doi:10.1016/S1384-1076(02)00193-8, 2003.

Shaviv, N. J., and J. Veizer, A celestial driver of phanerozoic climate?, GSA Today, pp. 4-11, 2003.

Siegenthaler, U., and F. Joos, Use of a simple model for studying oceanic tracer distributions and the global carbon cycle, Tellus, Series B-Chemical and Physical Meteorology, 44(3), 1992.

Soden, J. S., and I. M. Held, An assessment of climate feedbacks in coupled ocean-atmosphere models, J. Clim., 19, 3354-3360, 2006.

Solanki, S. K., and M. Fligge, Solar irradiance variations and climate, J. Atmos. Terr. Phys., 64, 677-685, 2002.

Svensmark, H., Influence of Cosmic Rays on Earth's Climate, Phys. Rev. Lett., 81, 5027-5030, 1998.

Svensmark, H., J. O. P. Pedersen, N. D. Marsh, M. B. Enghoff, and U. I. Uggerhoej, Experimental evidence for the role of ions in atmospheric particle nucleation, Proc. Roy. Soc. A., pp. 385-396, 2006.

van Loon, H., and K. Labitzke, The Influence of the 11-year Solar Cycle on the Stratosphere Below 30km: a Review, Sp. Sci. Rev., 94, 259-278, 2000.

White, W., J. Lean, D. Cayan, and M. Dettinger, Response of global upper ocean temperature to changing solar irradiance, J. Geophys. Res, 102(3), 255-3, 1997.

White, W. B., J. Lean, D. R. Cayan, and M. D. Dettinger, Response of global upper ocean temperature to changing solar irradiance, J. Geophys. Res., 102, 3255-3266, doi: 10.1029/96JC03549, 1997.

White, W. B., M. D. Dettinger, and D. R. Cayan, Global Average Upper Ocean Temperature Response to Changing Solar Irradiance: Exciting the Internal Decadal Mode, in ESA SP-463: The Solar Cycle and Terrestrial Climate, Solar and Space weather, edited by A. Wilson, p. 125, 2000.

Nir J. Shaviv, Racah Institute of Physics, Hebrew University of Jerusalem, Giv'at Ram, Jerusalem 91904, Israel (shaviv@phys.huji.ac.il) 